\title{
IMPROVEMENTS IN SPATIALLY RESOLVED CHARACTERIZATION USING EELS AND EFTEM SPECTRUM-IMAGE DATASETS
}

\author{
P.J. Thomas, C. Trevor, R.T. Harmon, M.K. Kundmann and J.A. Hunt \\ Gatan Research and Development, 5933 Coronado Lane, Pleasanton, CA 94588 USA
}

The ability to acquire routinely electron energy-loss spectrum-images over a range of typically hundreds of eV loss (or more) provides a powerful means for rapid materials characterisation via chemical mapping. Whether by recording a linear series of parallel spectra to form an EELS spectrum-image (EELS-SI), or by serially sampling energy-loss space through a series of energyfiltered images to build an EFTEM spectrum-image (EFTEM-SI), such datasets can contain information relating to multiple chemical species suitable for mapping. A number of analysis tools have been developed to optimize the extraction of such information specifically from spectrumimage datasets. These methods include Fourier deconvolution for the effective removal of plural scattering effects, Kramers-Kronig analysis for the calculation of absolute thickness maps, and multiple linear least-squares fitting (MLLS) of reference spectra for the effective separation of overlapping edges and weak signal extraction.

Figs. a-e show an example of the use of such tools in spectrum-image analysis. A scanning EELS-SI dataset was acquired from a p-SiLK semiconductor sample [1], prepared by focussed ion beam milling (FIB), and recorded using a Gatan ENFINA spectrometer attached to a JEOL 2010F (S)TEM [2]. The EELS-SI dataset was acquired over $200 \times 200$ pixels from $180 \mathrm{eV}$ to $1520 \mathrm{eV}$ with $8 \mathrm{~ms}$ dwell time per spectrum and $0.5 \mathrm{nA}$ beam current. A dark field image of the region analyzed is shown in Fig. a. The primary edges of interest for this data are the oxygen $\mathrm{K}$ and the chromium $\mathrm{L}_{23}$ edges at $532 \mathrm{eV}$ and $575 \mathrm{eV}$ respectively. The conventional power-law approach for generating elemental maps involves modeling, extrapolating and removing the characteristic background intensity for each pixel using a pre ionization-edge fitting region. However, as a direct consequence of the close proximity of the $\mathrm{O}$ and $\mathrm{Cr}$ edges, serious artifacts can result in the generated $\mathrm{Cr}$ distribution map since the post-edge features of the preceding oxygen edge can invalidate the use of this simple background model. The two elemental maps shown in Figs. b and c, computed using the power-law approach, illustrate this. While the oxygen map shows no obvious artifacts, the chromium map shows an unphysical band of highly negative counts where the preceding oxygen edge causes the background model to fail.

A more effective approach is to use MLLS fitting of reference spectra to determine accurately the relative contributions of the two overlapping edges [2]. After removing the pre-oxygen edge background signal using conventional power-law modeling, pre-acquired oxygen and chromium reference spectra were fitted on a pixel-by-pixel basis to yield concentration distribution maps. The resultant oxygen map (not shown) is almost indistinguishable from the corresponding power-law generated map. The MLLS computed chromium map, however, contains significantly less noise than its power-law counterpart, and does not show the dominant negative band artifact (Fig.d). This is illustrated effectively by comparison of compositional line traces from the MLLS and power-law generated maps (Fig. e); the MLLS computed line-trace gives realistic compositional values even in the $\mathrm{O}$ rich band, demonstrating the technique's ability to separate overlapping signals for mapping.

This and other examples will be presented to illustrate the effectiveness of the techniques developed for performing quantitative elemental mapping with spectrum-image datasets. 


\section{References:}

[1] DOW chemical company are gratefully acknowledged for provision of the sample used in this study.

[2] C. Trevor et al, Proceedings of Microscopy and Microanalysis (2001) 1148

[3] R.D. Leapman and C.R. Swyt, Ultramicroscopy 26 (1988) 393

a)

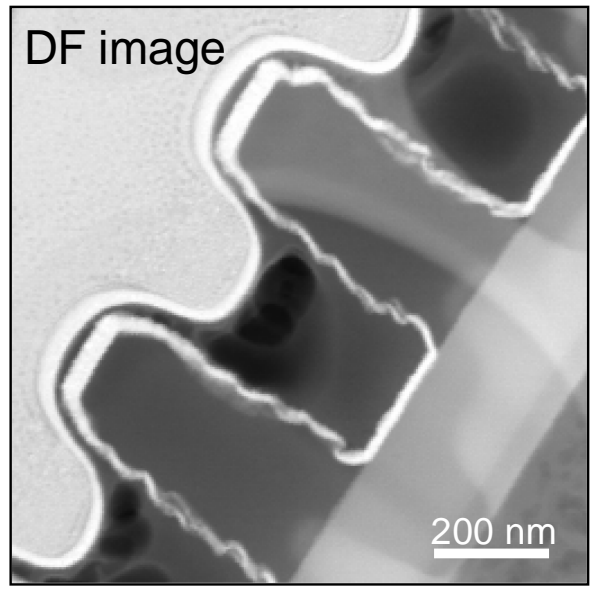

c)

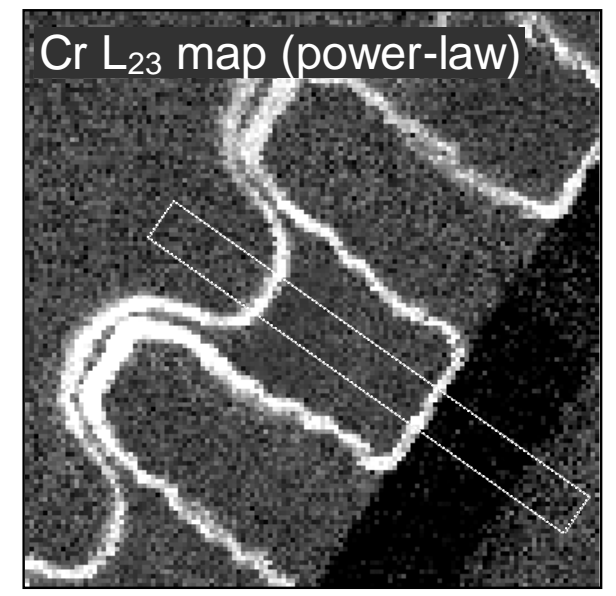

e)

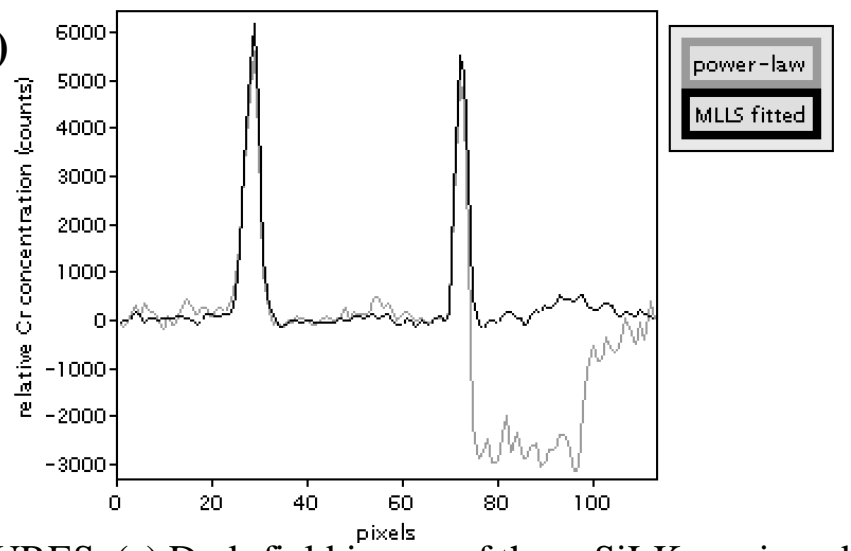

b)

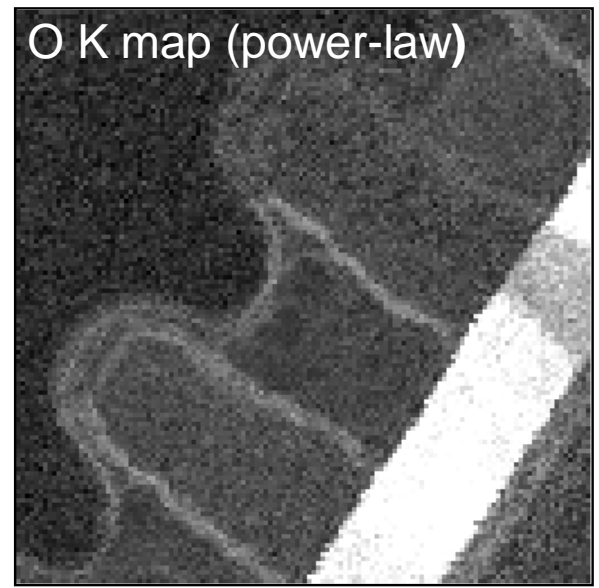

d)

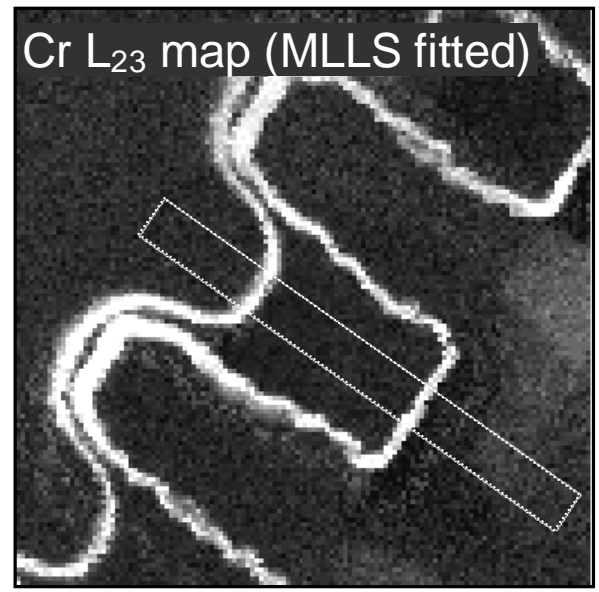

FIGURES: (a) Dark-field image of the p-SiLK semiconductor device. (b) O K map and (c) $\mathrm{Cr} \mathrm{L}_{23}$ elemental maps computed via power-law background removal. (d) $\mathrm{Cr} \mathrm{L}_{23}$ elemental map computed using MLLS fitting of reference spectra. (e) Comparison of compositional line traces taken from the regions indicated in (c) and (d) illustrate that the artifacts arising from background fit errors in the power-law generated Cr map (filled plot) are not present in the MLLS generated map. 